Pacific Journal of Mathematics

EXISTENCE OF HALF-TRAJECTORIES IN PRESCRIBED
REGIONS AND ASYMPTOTIC ORBITAL STABILITY 


\title{
EXISTENCE OF HALF-TRAJECTORIES IN PRESCRIBED REGIONS AND ASYMPTOTIC ORBITAL STABILITY
}

\author{
W. E. JOHNSON
}

\begin{abstract}
A theorem is proved concerning the existence of a halftrajectory in the neighborhood of a semi-invariant set of a general dynamical system. A corollary of this theorem strengthens a result of $P$. Mendelson. The theorem is further used to obtain a necessary and sufficient condition for a compact positively invariant set to be positively asymptotically orbitally stable, and the condition is compared with another one due to S. Lefschetz.
\end{abstract}

Mendelson [3] applied a topological method due to Wazewski to obtain a sufficient condition for a neighborhood of a rest point of an autonomous system of differential equations to contain a half trajectory other than the rest point. The condition is that all points of egress be points of strict egress. Actually, as we show in $\S 2$, this condition is redundant. Any neighborhood of a rest point of a dynamical system defined on an open set in $E^{n}$ contains a half trajectory other than the rest point (possibly another rest point).

The purpose of this paper is twofold. First, in $\S 2$ we prove a theorem on the existence of a half trajectory in the neighborhood of a semi-invariant set of a general dynamical system and from it deduce three corollaries. Corollary 2 is a generalization of Mendelson's theorem ([3], p. 221). Corollary 3 gives a sufficient condition for a neighborhood of a rest point to contain a half trajectory which is not a rest point, and it is shown by example that, in a sense, the condition is the best possible. Second, in $\S 3$ Theorem 1 is used to obtain a necessary and sufficient condition for a compact positively invariant set to be positively asymptotically orbitally stable. This condition is compared with another one due to Lefschetz [2].

An autonomous system of differential equations fails to generate a dynamical system in the sense of Nemytskii-Stepanov [5, part II] if the solutions do not exist for all values of the independent variable. However, although the present discussion pertains to a dynamical system, with minor modifications the results in $\S 2$ can be extended to autonomous systems of differential equations whose right hand sides

Received December 7, 1964. This research was supported by the Lockheed Independent Research Program. 
are continuous and whose solutions are uniquely determined by their initial values.

2. Existence of half trajectories in a neighborhood of a semi-invariant set. Let $R$ be a metric space with distance function $\rho$, and denote the closure, complement, and boundary of a set $A \subset R$ by $\bar{A}, A^{\prime}$, and $\partial A$ respectively. The open $\varepsilon$-sphere about either a point $A \in R$ or a set $A \subset R$ will be written $S(A, \varepsilon)=\{p \in R: \rho(p, A)<\varepsilon\}$. We denote the real intervals $(-\infty, \infty),(-\infty, 0]$ and $[0, \infty)$ by $I, I^{-}$, and $I^{+}$respectively. Let $f$ be a dynamical system on $R$, i.e., $f$ is a continuous transformation on $R \times I$ onto $R$ such that $f(p, 0)=p$ and $f\left(f\left(p, t^{\prime}\right), t\right)=f\left(p, t^{\prime}+t\right)$ for all $p \in R, t, t^{\prime} \in I$. The sets $f\left(p, I^{\dagger}\right)$ and $f\left(p, I^{-}\right)$, for any $p \in R$, are called (positive and negative) half trajectories. The $\alpha$ - and $\omega$ - limit sets of a motion $f(p, t)$ will be denoted by $A_{p}$ and $\Omega_{p}$ respectively. A nonempty set $A \subset R$ is said to be positively (negatively) invariant if $f(A, t) \subset A$ for all $t \geqq 0(t \leqq 0)$, invariant if $f(A, I)=A$.

THEOREM 1. Let $F$ be a closed positively (negatively) invariant set and let $G$ be an open set which contains $F$ and has a compact boundary. If there exist $p_{n} \in G, p_{0} \in F$, and $t_{n} \in I, n=1,2, \cdots$, such that $p_{n} \rightarrow p_{0}, t_{n}>0\left(t_{n}<0\right)$, and $f\left(p_{n}, t_{n}\right) \in G^{\prime}$, then $\bar{G} \cap F^{\prime}$ contains a negative (positive) half trajectory.

Proof. ${ }^{\perp}$ We give the proof for the case in which $F$ is positively invariant. It follows from the hypothesis and well known properties of dynamical systems that there exist $T_{n}>0$ such that $f\left(p_{n}, t\right) \in G$ if $0 \leqq t<T_{n}, f\left(p_{n}, T_{n}\right) \in \partial G$ and $T_{n} \rightarrow \infty$. Let $q_{n}=f\left(p_{n}, T_{n}\right)$. Since $\partial G$ is compact, $\left\{q_{n}\right\}$ contains a convergent subsequence, and we can assume $q_{n} \rightarrow q_{0} \in \partial G$. The inclusion $F \subset G$ implies $q_{0} \notin F$, and it follows from the positive invariance of $F$ that $f\left(q_{0}, I^{-}\right) \cap F=\varnothing$. Given any $\tau<0$, $f\left(p_{n}, T_{n}+\tau\right)=f\left(q_{n}, \tau\right) \rightarrow f\left(q_{0}, \tau\right)$. Since $T_{n} \rightarrow \infty, 0 \leqq T_{n}+\tau<T_{n}$ whence $f\left(p_{n}, T_{n}+\tau\right) \in G$ for $n$ sufficiently large. Therefore $f\left(q_{0}, \tau\right) \in \bar{G}$ for all $\tau \leqq 0$.

CoRollary 1. Given a closed positively or negatively invariant set $F$, let $H=f(F, I)$. If $(\partial H) \cap F \neq \varnothing$, and if $G$ is an open set which contains $F$ and has a compact boundary, then $\bar{G} \cap F^{\prime}$ contains a half trajectory.

Proof. Let $F$ be positively invariant and suppose $\bar{G} \cap F^{\prime}$ contains no positive half trajectory. Choose $p_{0} \in F \cap(\partial H)$. Then there exist $p_{n} \in G \cap H^{\prime}$ such that $p_{n} \rightarrow p_{0}$, and it follows from the invariance of

\footnotetext{
${ }^{1} \mathrm{I}$ am indebted to the referee for pointing out a simplification of this proof.
} 
$H$ that $f\left(p_{n}, I^{+}\right) \cap F=\varnothing$. Hence, by assumption, there exist $t_{n}>0$ such that $f\left(p_{n}, t_{n}\right) \in G^{\prime}$, and it follows from Theorem 1 that $\bar{G} \cap F^{\prime}$ contains a negative half trajectory.

A simple example shows that the hypothesis $(\partial H) \cap F \neq \varnothing$ is essential to Corollary 1 . Let $R$ be the closed unit interval, and consider a dynamical system which has rest points at 0 and 1 and for which the motion through any point in the open interval $(0,1)$ has $\{0\}$ as its $\alpha$ limit set and $\{1\}$ as its $\omega$-limit set. If $F=[1 / 2,1]$ and $G=(1 / 4,1]$, then $H=(0,1]$ and $(\partial H) \cap F=\{0\} \cap[1 / 2,1]=\varnothing$. The rest of the hypothesis of Corollary 1 is fulfilled but the conclusion fails to hold.

CoROLlaRY 2. If $R$ is locally compact, if $F$ is a compact positively or negatively invariant set such that $(\partial H) \cap F \neq \varnothing$ where $H=f(F, I)$, and if $G$ is an open set which contains $F$, then $G \cap F^{\prime}$ contains a half trajectory whose closure is compact.

Proof. Since $R$ is locally compact and $F$ is compact, there exists an open set $U$ such that $F \subset U, \bar{U} \subset G$, and $\bar{U}$ is compact. By corollary $1, F^{\prime} \cap \bar{U}$ contains a half trajectory; its closure is contained in $\bar{U}$ and is therefore compact.

CoRollary 3. If $R$ is connected, if $G$ is an open set which contains a rest point, and if $\partial G$ is compact, nonempty and contains no rest point, then $\bar{G}$ contains a half trajectory which is not a rest point.

Proof. Let $F$ be the set of all rest points in $G$; obviously $F$ is invariant. Since the set of all rest points in $R$ is closed and disjoint from $\partial G, F$ is closed. Since $R$ is connected and $\partial G \neq \varnothing, F \cap(\partial F) \neq \varnothing$, and the result follows from corollary 1 .

Corollaries 1 and 2 are valid if $\partial G=\varnothing$. This follows from the fact that any finite are $f\left(p,\left[\tau_{1}, \tau_{2}\right]\right)$ is connected and so if $\partial G=\varnothing$, $f(p, I) \subset G$ for all $p \in G$. Thus if $(\partial H) \cap F \neq \varnothing$, there exists $p \in G \cap F^{\prime}$ and it follows from the one-side invariance of $F$ that either $f\left(p, I^{+}\right) \subset F^{\prime}$ or $f\left(p, I^{-}\right) \subset F^{\prime}$. However, the hypothesis $\partial G \neq \varnothing$ is essential to Corollary 3. For example, let every point of $R$ be a rest point and take $G=R$. An example which shows that Corollary 3 is not valid if $\partial G$ is permitted to have even one rest point is the dynamical system in the plane determined by the autonomous system of differential equations 


$$
\begin{gathered}
\dot{x}=y \\
\dot{y}=\left\{\begin{aligned}
-x & \text { if } \quad x<0 \\
0 & \text { if } x \geqq 0 .
\end{aligned}\right.
\end{gathered}
$$

The semi-axis $y=0, x \geqq 0$ consists of rest points. All other motions $x(t), y(t)$ traverse semi-circles above the origin in the left half plane and $x(t) \rightarrow \infty$ as $t \rightarrow \infty$ and as $t \rightarrow-\infty$. Thus the boundary of any sphere about the origin contains precisely one rest point, but no such sphere contains a half trajectory which is not a rest point.

3. Asymptotic orbital stability. A compact positively invariant set $F$ is said to be positively orbitally stable is given $\varepsilon>0$ there exists $\delta>0$ such that for all $q \in S(F, \delta), f\left(q, I^{+}\right) \subset S(F, \varepsilon)$. Because of the compactness of $F$, this is equivalent to the requirement that given $\varepsilon>0$ and $p \in F$, there exist $\delta>0$ such that for all $q \in S(p, \delta)$, $f\left(q, I^{+}\right) \subset S(F, \varepsilon)$. A compact positively invariant set $F$ is said to be positively asymptotically orbitally stable if it is positively orbitally stable and if there exists $\delta^{\prime}>0$ such that for all $q \in S\left(F, \delta^{\prime}\right), \Omega_{q} \subset F$. This implies that $f(q, t)$ is positively Lagrange stable for all $q \in S\left(F, \delta^{\prime}\right)$, and it is a consequence of [5, Theorem 3.07, p. 341] that the condition $\Omega_{q} \subset F$ is equivalent to the more usual condition $\lim _{t \rightarrow \infty} \rho(f(q, t), F)=00^{1}$ These definitions of stability agree with the terminology of AuslanderSeibert [1] and Lefschetz [2] except that they omit the prefix "orbital." The qualifier will be retained here in order to avoid confusion with other types of stability and because it conforms with the terminology usually applied to limit cycles. A necessary condition" for $F$ to be positively orbitally stable is that $A_{q} \cap F=\varnothing$ for all $q \in F^{\prime}$. For if $q \in F^{\prime}$ and $p \in A_{q} \cap F$, then there exist $t_{n}<0$ such that $t_{n} \rightarrow-\infty$ and $q_{n}=f\left(q, t_{n}\right) \rightarrow p$. Since $F$ is closed, $\rho(q, F)=\varepsilon>0$. Hence given any $\delta>0$, there exists $n$ for which $q_{n} \in S(p, \delta)$ and $\rho\left(f\left(q_{n},-t_{n}\right), F\right)=$ $\rho(q, F)=\varepsilon$. Therefore $F$ is not positively orbitally stable. That $F$ can fail to be positively orbitally stable in precisely this way is indicated in [4], where there is sketched (Figure 1) a dynamical system in the plane for which the origin 0 is a rest point and $A_{q}=\Omega_{q}=\{0\}$ for all $q \in R$. In the next theorem we show that the requirement $A_{q} \cap F=\varnothing$ for $q \in F^{\prime}$ together with a weaker requirement than $\Omega_{q} \subset F$ for $q$ in some neighborhood of $F$ constitute a necessary and sufficient condition for positive asymptotic orbital stability. Because of the compactness of $F$, any open set containing $F$ contains $S(F, \varepsilon)$ for some $\varepsilon>0$, and therefore it is only a matter of notational convenience to

1 This is also observed [1] p. 459.

2 This condition is explicitly stated in $[2,(15.2)]$ for the case where $F$ is a rest point of an autonomous system of differential equations. 
make use of arbitrary open sets containing $F$ rather than sets of the specific form $S(F, \varepsilon)$.

THEOREM 2. Let $F$ be a compact positively invariant set which is contained in an open set $G$ with compact closure. A necessary and sufficient condition for $F$ to be positively asymptotically orbitally stable is that there exist an open set $G_{1}$ containing $F$ such that $A_{q} \cap F=\varnothing$ and $\Omega_{q} \cap F \neq \varnothing$ for all $q \in \bar{G}_{1} \cap F^{\prime}$.

Proof. In view of the remark in the preceding paragraph, the necessity is obvious. To prove the sufficiency we first show that $F$ is positively orbitally stable. Assuming the contrary we obtain $p \in F$, $p_{n} \in G, t_{n}>0$ and $\varepsilon>0$ such that $p_{n} \rightarrow p$ and $\rho\left(f\left(p_{n}, t_{n}\right), F\right) \geqq \varepsilon$. Thus, letting $G_{2}=S(F, \varepsilon) \cap G_{1} \cap G, f\left(p_{n}, t_{n}\right) \in G_{2}^{\prime}$ and it follows from Theorem 1 (note that $p_{n} \in G_{2}$ for $n$ sufficiently large) that there exists $q \in \bar{G}_{2}$ such that $f\left(q, I^{-}\right) \subset \bar{G}_{2} \cap F^{\prime}$. Since $\bar{G}_{2}$ is compact, $A_{q} \neq \varnothing$, say $q_{1} \in A_{q}$, and $A_{q} \subset \bar{G}_{2}$. Since $A_{q}$ is closed and invariant, $\Omega_{q_{1}} \subset A_{q}$; by hypothesis, $\Omega_{q_{1}} \cap F \neq \varnothing$ and so $A_{q} \cap F \neq \varnothing$. But this contradicts the other part of the hypothesis and therefore $F$ is positively orbitally stable.

Now assume $F$ is not positively asymptotically orbitally stable. Then there exists $q \in G_{1}$ such that $\Omega_{q} \cap F^{\prime} \neq \varnothing \varnothing$ and, by hypothesis, $\Omega_{q} \cap F \neq \varnothing$. Choose $p_{0} \in \Omega_{q} \cap F, q_{0} \in \Omega_{q} \cap F^{\prime}$, and let $\rho\left(q_{0}, F\right)=\varepsilon$. Then $\varepsilon>0$ and, given any $\hat{o}>0$, there exist $t>0$ and $t^{\prime}>t$ such that $f(q, t) \in S\left(p_{0}, \delta\right)$ and $f\left(q, t^{\prime}\right) \in S\left(q_{0}, \varepsilon / 2\right)$. Hence

$$
f\left(q, t^{\prime}\right)=f\left(f(q, t), t^{\prime}-t\right) \notin S\left(F, \frac{\varepsilon}{2}\right)
$$

and $t^{\prime}-t>0$. This contradicts the positive orbital stability of $F$ and the proof is complete.

Another set of necessary and sufficient condition for positive asymptotic orbital stability of an invariant set $F$ is given in [2, (14.3)]. These conditions comprise a necessary and sufficient condition for positive orbital stability together with the requirement that there exist a neighborhood of $F$ which contains no entire trajectory other than those in $F$. On the other hand, no part of the hypothesis of Theorem 2 is both a necessary and sufficient condition for positive orbital stability alone, and in this respect the two results are essentially different. 


\section{REFERENCES}

1. J. Auslander, and P. Seibert, Prolongations and Generalized Liapunov Functions, International Symposium on Nonlinear Differential Equations and Nonlinear $\mathrm{Me}$ chanics, Academic Press, (1963), 454-462.

2. S. Lefschetz, Liapunov and Stability in Dynamical Systems, Bol. Soc. Mat. Mex., [2], 3, (1958), 25-39.

3. P. Mendelson, On Lagrange Stable Motions in the Neighborhood of Critical Points, Contributions to the Theory of Nonlinear Oscillations, Vol. V, Annals of Mathematics Studies No. 45, Princeton University Press, (1960), 219-224.

4. - On Unstable Attractors, Bol. Soc. Mat. Mex. [2], 5, (1960), 270-276.

5. V. V. Nemytskii, and V. V. Stepanov, Qualitative Theory of Differential Equations, Princeton University Press, 1960.

Lockheed Missile \& SpaCe Company

Palo Alto, California 


\section{PACIFIC JOURNAL OF MATHEMATICS}

\section{EDITORS}

\author{
H. SAMELSON \\ Stanford University \\ Stanford, California \\ R. M. BLUMENTHAL \\ University of Washington \\ Seattle, Washington 98105
}

\author{
*J. DUGUNDJI \\ University of Southern California \\ Los Angeles, California 90007
}

RICHARD ARENS

University of California Los Angeles, California 90024

\section{ASSOCIATE EDITORS}
E. F. BECKENBACH
B. H. NEUMANN
F. WOLF
K. YoSIDA

\section{SUPPORTING INSTITUTIONS}

\author{
UNIVERSITY OF BRITISH COLUMBIA \\ CALIFORNIA INSTITUTE OF TECHNOLOGY \\ UNIVERSITY OF CALIFORNIA \\ MONTANA STATE UNIVERSITY \\ UNIVERSITY OF NEVADA \\ NEW MEXICO STATE UNIVERSITY \\ OREGON STATE UNIVERSITY \\ UNIVERSITY OF OREGON \\ OSAKA UNIVERSITY \\ UNIVERSITY OF SOUTHERN CALIFORNIA
}

\author{
STANFORD UNIVERSITY \\ UNIVERSITY OF TOKYO \\ UNIVERSITY OF UTAH \\ WASHINGTON STATE UNIVERSITY \\ UNIVERSITY OF WASHINGTON \\ * * * * \\ AMERICAN MATHEMATICAL SOCIETY \\ CHEVRON RESEARCH CORPORATION \\ TRW SYSTEMS \\ NAVAL ORDNANCE TEST STATION
}

Mathematical papers intended for publication in the Pacific Journal of Mathematics should be typewritten (double spaced). The first paragraph or two must be capable of being used separately as a synopsis of the entire paper. It should not contain references to the bibliography. Manuscripts may be sent to any one of the four editors. All other communications to the editors should be addressed to the managing editor, Richard Arens at the University of California, Los Angeles, California 90024.

50 reprints per author of each article are furnished free of charge; additional copies may be obtained at cost in multiples of 50 .

The Pacific Journal of Mathematics is published monthly. Effective with Volume 16 the price per volume (3 numbers) is $\$ 8.00$; single issues, $\$ 3.00$. Special price for current issues to individual faculty members of supporting institutions and to individual members of the American Mathematical Society: $\$ 4.00$ per volume; single issues $\$ 1.50$. Back numbers are available.

Subscriptions, orders for back numbers, and changes of address should be sent to Pacific Journal of Mathematics, 103 Highland Boulevard, Berkeley 8, California.

Printed at Kokusai Bunken Insatsusha (International Academic Printing Co., Ltd.), No. 6, 2-chome, Fujimi-cho, Chiyoda-ku, Tokyo, Japan.

\section{PUBLISHED BY PACIFIC JOURNAL OF MATHEMATICS, A NON-PROFIT CORPORATION}

The Supporting Institutions listed above contribute to the cost of publication of this Journal, but they are not owners or publishers and have no responsibility for its content or policies.

* Paul A. White, Acting Editor until J. Dugundji returns. 


\section{Pacific Journal of Mathematics}

\section{Vol. 17, No. 2 \\ February, 1966}

Henry A. Antosiewicz, Boundary value problems for nonlinear ordinary

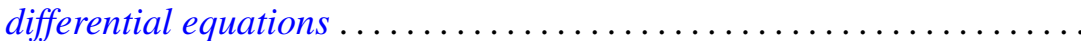

Bernard Werner Levinger and Richard Steven Varga, Minimal Gerschgorin sets. II ........................................

Paul Camion and Alan Jerome Hoffman, On the nonsingularity of complex matrices........................................ 211

J. Chidambaraswamy, Divisibility properties of certain factorials ........ 215

J. Chidambaraswamy, A problem complementary to a problem of Erdös .... 227

John Dauns, Chains of modules with completely reducible quotients ...... 235

Wallace E. Johnson, Existence of half-trajectories in prescribed regions and asymptotic orbital stability ............................ 243

Victor Klee, Paths on polyhedra. II . ........................ 249

Edwin Haena Mookini, Sufficient conditions for an optimal control problem in the calculus of variations ............................ 263

Zane Clinton Motteler, Existence theorems for certain quasi-linear elliptic equations........................................... 279

David Lewis Outcalt, Simple n-associative rings ............... 301

David Joseph Rodabaugh, Some new results on simple algebras ......... 311

Oscar S. Rothaus, Asymptotic properties of groups generation ........... 319

Ernest Edward Shult, Nilpotence of the commutator subgroup in groups admitting fixed point free operator groups .............

William Hall Sills, On absolutely continuous functions and the

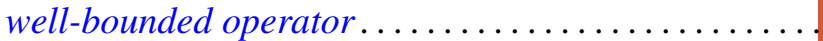

Joseph Gail Stampfli, Which weighted shifts are subnormal ..

Donald Reginald Traylor, Metrizability and completeness in normal Moore spaces 